\title{
Patient Perspectives on Self-Management Technologies for Chronic Fatigue Syndrome
}

\author{
Tabby Davies \\ University of Bath \\ Bath, United Kingdom \\ tjpd20@bath.ac.uk
}

\author{
Simon L. Jones \\ University of Bath \\ Bath, United Kingdom \\ s.l.jones@bath.ac.uk
}

\author{
Ryan M. Kelly \\ The University of Melbourne \\ Melbourne, Australia \\ ryan.kelly@unimelb.edu.au
}

\begin{abstract}
Chronic Fatigue Syndrome (CFS) is a debilitating medical condition that is characterized by a range of physical, cognitive and social impairments. This paper investigates CFS patients' perspectives on the potential for technological support for self-management of their symptoms. We report findings from three studies in which people living with CFS 1) prioritized symptoms that they would like technologies to address, 2) articulated their current approaches to self-management alongside challenges they face, and 3) reflected on their experiences with three commercial smartphone apps related to symptom management. We contribute an understanding of the specific needs of the ME/CFS population and the ways in which they currently engage in self-management using technology. The paper ends by describing five high-level design recommendations for ME/CFS self-management technologies.
\end{abstract}

\section{CCS CONCEPTS}

- Human-centered computing $\rightarrow$ Empirical studies in HCI.

\section{KEYWORDS}

Chronic Fatigue Syndrome; Myalgic Encephalomyelitis; selfmanagement; self-tracking.

ACM Reference Format:

Tabby Davies, Simon L. Jones, and Ryan M. Kelly. 2019. Patient Perspectives on Self-Management Technologies for Chronic Fatigue Syndrome. In CHI Conference on Human Factors in Computing Systems Proceedings (CHI 2019), May 4-9, 2019, Glasgow, Scotland UK. ACM, New York, NY, USA, 13 pages. https://doi.org/10.1145/3290605. 3300452

\section{INTRODUCTION}

Research in HCI has begun to explore the potential for technologies to assist with the self-management of chronic health

CHI 2019, May 4-9, 2019, Glasgow, Scotland UK

(C) 2019 Copyright held by the owner/author(s). Publication rights licensed to ACM.

This is the author's version of the work. It is posted here for your personal use. Not for redistribution. The definitive Version of Record was published in CHI Conference on Human Factors in Computing Systems Proceedings (CHI 2019), May 4-9, 2019, Glasgow, Scotland UK, https://doi.org/10.1145/3290605.3300452. conditions. In this context, self-management refers to an individual's ability to "manage the symptoms, treatment, physical and psychosocial consequences and lifestyle changes inherent to living with a chronic condition" [7, p. 178]. Previous work suggests that technology can support self-management by promoting reflective thinking [46], helping to monitor symptom triggers [10], and by gathering evidence to facilitate deeper understanding of a condition [27]. Examples of conditions studied by HCI researchers include chronic pain [3], HIV+ [11], migraine [65] and irritable bowel syndrome [66].

In this paper we focus on the design of self-management technologies for people living with Chronic Fatigue Syndrome, or Myalgic Encephalomyelitis (ME/CFS) ${ }^{1}$. ME/CFS is an example of an enigmatic disease [48] in that it has a range of symptoms, little clinical consensus on effective treatment [75], and a lack of symptom specificity. While ME/CFS is thought to affect $0.2-0.4 \%$ of the world's population $[26,32,36]$, research indicates that up to $90 \%$ of cases go undiagnosed [32], suggesting that people with ME/CFS are substantially undercounted and undertreated [19]. This is problematic because the condition has an array of debilitating symptoms, including extreme tiredness following exertion, cognitive fatigue, rheumatic pain and disrupted sleep. These symptoms impact quality of life [39] and can make the condition very difficult to manage for patients. Furthermore, many people with ME/CFS report that clinicians do not always provide empathetic support [41], which may be due to a lack of clinical consensus around proper treatment of the disease [74,75].

Our work aims to support people with ME/CFS by exploring the ways in which digital technologies should support self-management of the condition. While self-management can be achieved through the use of generic solutions (e.g. smartphone apps [38]), previous work has demonstrated the need to design around the symptoms of a given condition and promote self-care in light of those symptoms $[11,56,58,59]$. However, designers may struggle to create tailored support for ME/CFS without a clear understanding of patients' needs

\footnotetext{
${ }^{1}$ We use the abbreviation ME/CFS as this follows the convention used in recent scholarly literature [e.g. 19, 20, 29, 75] and because some patients feel that the phrase 'chronic fatigue syndrome' is a misnomer that trivializes the medical nature of the condition [21].
} 
and goals for self-management. Furthermore, while the prevalence of particular symptoms associated with ME/CFS is well understood $[28,71]$ there is a lack of knowledge about which symptoms patients want to manage the most.

Our work addresses these gaps by adopting a patient-centric approach in which we engage with ME/CFS patients to learn about their perspectives on the potential for self-management technologies. We provide three contributions for supporting the design of self-management technologies for ME/CFS. First, we provide a list of symptoms prioritized by people with ME/CFS, giving immediate direction for the design of condition-specific support. Second, we find that people with $\mathrm{ME} / \mathrm{CFS}$ engage in self-management through off-the-shelf technologies that do not provide effective support for their condition. Finally, participants' responses to a series of commercial apps reveal positive and negative experiences with specific features. From our studies we derive five recommendations that can guide the design of future self-management technologies for people living with ME/CFS.

\section{BACKGROUND}

ME/CFS is a chronic (i.e. long-term) illness with an unknown aetiology [31]. The primary clinical feature of the condition is a severe disabling fatigue that has been present for at least six consecutive months [28]. ME/CFS has a complex array of symptoms. The foremost of these is post-exertional malaise (PEM), which refers to symptoms that arise following mental or physical exertion $[19,35]$. These symptoms include a loss of stamina and intense tiredness that can only be alleviated through long periods of rest [72]. PEM is highly debilitating and can occur after even the simplest of tasks, including "walking, showering, or having a conversation" [35, p. 20].

$\mathrm{ME} / \mathrm{CFS}$ is also characterized by neurocognitive impairment (known as "brain fog" [57]) that impacts memory, concentration and problem-solving ability [67]. Other recognized symptoms include rheumatic pain affecting the muscles and joints $[1,4]$; poor quality of sleep, which typically leaves individuals feeling unrefreshed and thus prolongs feelings of tiredness [4, 67]; and flu-like symptoms including sore throat and headaches [32]. ME/CFS patients can experience these symptoms with different degrees of severity. Some are affected so heavily that they are left bedridden and dependent on others for care $[4,72,76]$. Others may lead a relatively normal life that is intermittently disrupted by fatigue that lasts for several days or weeks before subsiding [39]. Symptoms can also vary on a daily basis, with patients reporting both "good days and bad days" [39, p. 9].

The highly disabling nature of ME/CFS impacts both physical and mental health. Patients' lack of physical activity can result in muscle deconditioning, making movement even harder [4]. Around $66 \%$ of people with ME/CFS meet clinical criteria for depression [2] and up to $85 \%$ of patients cite depressed mood as a major symptom [39, 43]. Other research indicates that people with ME/CFS suffer from perpetuating factors including mood disturbance, kinesiophobia (a fear of movement [54]), and low self-efficacy [71]. These factors can lead to a perceived lack of independence and a reduction in social life [76]. Some patients struggle to accept the realities of the condition and exhibit a tendency to imagine the worst (known as 'catastrophizing') [71].

\section{Self-Management for ME/CFS}

Several formalized programs have been promoted as evidencebased treatments for ME/CFS [29]. These include graded exercise therapy, a form of physical therapy where physical activity is gradually increased over time [24], and cognitive behavioral therapy, which aims to improve mental health by addressing the prevalence of negative thoughts and behaviors [61].

While early studies indicated that these programs may help to alleviate the symptoms of ME/CFS [e.g. 60], recent research has argued that there is limited evidence of their effectiveness $[70,74,75]$. In addition, participating in these courses can be very difficult because they require attendance at in-person treatment sessions. This is problematic because instances of PEM are associated with changes in routine, prolonged cognitive effort, and travelling [40]. Individuals with ME/CFS have reported that their symptoms worsen as a result of participating in such programs [29] and thus many receive no formal treatment for their condition [32]. ME support organizations such as Emerge [25] consequently provide guidance for patients who choose to engage in self-management practices and manage the condition by themselves [25].

A primary form of self-management for ME/CFS is activity pacing [30]. The goal of activity pacing is to manage one's energy expenditure and avoid PEM by working within one's limits [29]. This is done through the planning and routinization of day-to-day activities [30], maintaining consistency in activity levels, accepting current abilities, and gradually increasing activities over time [6]. Common approaches to activity pacing include staying within an 'energy envelope' [33], which involves balancing energy expenditure so as not to under- or over-exert oneself, and 'spoon management' [50], which involves imagining one's energy as a limited currency that is 'spent' on daily tasks. For example, having a shower would 'cost' one spoon of energy. Other self-management activities for ME/CFS include mindfulness techniques [68], which help to reduce stress and create inner calm, and the setting of meaningful and realistic activity goals in order to avoid over-exertion [6]. However, little is known about how people with ME/CFS currently use digital technologies to pursue self-management practices, nor how these technologies should be designed in light of what these practices entail. 


\section{RESEARCH OVERVIEW}

The studies we report in this paper shed light on the current technologically-mediated self-management practices of people living with ME/CFS, providing knowledge of how technologies should be designed to better support those practices. We build on the approach of McKillop et al. [48] by triangulating multiple sources of data to understand patients' current approaches to managing an enigmatic disease. The data sources we utilize comprise surveys to identify priorities for self-management of ME/CFS (Study 1); online discussions of existing ME/CFS self-management practices and technology use (Study 2); and user evaluations of three smartphone apps that address some of these priorities (Study 3).

Study 1 shows which symptoms people with ME/CFS wish to prioritize, but does not provide insight into how this might be done. Study 2 addresses this by investigating how people currently self-manage using technologies, and reveals difficulties they encounter, giving direction for design. Study 3 then probes existing apps that enable management of symptoms identified in Study 1 and practices highlighted in Study 2, revealing additional design-relevant issues. The three studies provide complementary perspectives on the research problem and contribute to a set of design implications and recommendations, listed at the end of the paper.

\section{Recruitment and Ethical Considerations}

One of the immediate challenges of conducting research with $\mathrm{ME} / \mathrm{CFS}$ patients is that it is unclear how their involvement might exacerbate the symptoms of their condition. As noted above, some patients can suffer serious consequences when deviating from set routines [40] and thus participation in a research study could cause undue harm by inducing PEM, for example. This presents major challenges in terms of configuring their involvement, not just from a practical perspective (what techniques should be employed to involve patients?) but also from an ethical standpoint (how do we involve people with ME/CFS without exacerbating their symptoms?). As an example, the use of face-to-face design workshops (a well-established method in $\mathrm{HCI}[22,51,52,64]$ ) would require participants to attend sessions in-person, risking exacerbation of symptoms. Furthermore, this could rule out participation from those experiencing the most severe symptoms.

For this research, we elected to employ remote methods for all three of our studies as a way of accommodating the needs and challenges faced by people living with ME/CFS. Our primary means of recruitment was to approach people through support groups on Facebook and an online forum dedicated to ME/CFS. One of the researchers has personal experience of the condition and was an active contributor to these groups for several months before the research began. This experience supported the identification of suitable online communities to approach, allowed the researcher to empathize with the concerns of participants, and supported interpretation of responses during qualitative analysis.

Data collection and communication for all three studies took place via email, Facebook and discussion forums. This enabled participants to read and reply at their own convenience, and hence accommodated potential fluctuations in their illness while lowering the risk of harm. In addition, we conducted a full review of the ethical implications of our research prior to each study, following the institutional concordat for research integrity at the University of Bath. Our ethical review was approved by the local Departmental Research Ethics Officer. No financial incentives were offered to participants.

Our initial interactions with ME/CFS patients confirmed that many anticipated difficulty with participating in any research activities that might disrupt their typical routines or demand significant physical or mental exertion. Many expressed these limitations upfront. However, even participants with severe symptoms (e.g. being frequently housebound or experiencing extreme 'crashes') volunteered to take part. We made it clear to all participants that their involvement was voluntary and that they were free to withdraw or suspend at any time without consequence. We also provided flexibility of deadlines for completing the activities.

Early data collection suggested that participants would benefit from regular reminders to complete the requested tasks, with some stating that they often felt so overcome by their illness that they have difficulty remembering tasks and appointments. For this reason we issued frequent reminders to participants throughout each study, e.g. by revisiting message threads to invite further participation, or by sending gentle email reminders to people who had already consented to participate. We also took extra care in the design of our procedures by providing written (rather than verbal) instructions together with clear, actionable guidelines. We did this because it seemed likely that participants would need to revisit these when resuming their involvement, given their reported tendency to forget about the research.

While some of these steps may seem trivial and can be taken for granted in many research studies, we see them as critical for enabling the participation of ME/CFS patients. We found that participants appreciated the regular reminders and took advantage of the ability to respond flexibly in accordance with changes in their symptoms. Many were enthusiastic about being able to take part in the research.

\section{STUDY 1 - SURVEY: PRIORITIZING SYMPTOMS FOR ME/CFS SELF-MANAGEMENT}

Our first study seeks to identify the dimensions of ME/CFS that are most important for self-management. Previous work has established the prevalence of ME/CFS symptoms [39, 71] 
but has not focused on prioritizing these symptoms for selfmanagement technologies. We utilize a ranking task to investigate the self-management priorities that people with $\mathrm{ME} / \mathrm{CFS}$ assign to symptoms associated with the disease.

We compiled a list of 16 primary symptoms associated with the disease by combining the items from existing ME/CFS symptom surveys with our own literature review $[4,39,67,71$, $72]$. We then created an online survey that asked participants to rank the 16 symptom items provided in the list. Specifically, respondents were asked: "Which symptoms of your disease would you most like ME/CFS self-management apps to support?". The survey was advertised to members of an international $\mathrm{ME} / \mathrm{CFS}$ online discussion forum and a closed ME/CFS support group on Facebook.

We received responses from 39 people, 38 of whom had received a formal diagnosis of $\mathrm{ME} / \mathrm{CFS}$ from a clinician. One person was self-diagnosed. Participants' average age was 40.3yrs (Range $=18-64, \mathrm{SD}=9.8 \mathrm{yrs}$ ). 34 participants identified as female and 5 identified as male ${ }^{2} .25$ participants were residents of the United Kingdom; 9 USA; 2 Australia; 2 Canada, and 1 Norway. All participants were fluent English speakers.

\section{STUDY 1 FINDINGS}

Survey responses were analyzed by calculating the median ranking for each symptom using ratings from all 39 participants. Figure 1 shows the combined ratings for each of the symptoms. The boxplot indicates the median, upper quartile, lower quartile, and range of priority scores for the ratings received (on a normalized scale from $0-1$, where $0=$ lowest and 1 = highest ranking).

The results of the ranking exercise reveal several groups of symptoms that participants believed self-management technologies for ME/CFS should support. The highest priority scores were assigned to symptoms associated with physical fatigue (severe disabling fatigue, post-exertional malaise), cognitive fatigue and impairment (impaired memory and concentration), sleep-related symptoms (unrefreshing and disturbed sleep), and other physical side-effects (such as physical deconditioning and aching, weak or stiff muscles). Slightly lower priorities are given to managing aspects related to social wellbeing (lack of independence, reduction in social life), and emotional/affective responses to the disease (mood disturbance, lack of acceptance, low self-efficacy, introversion, catastrophizing).

While previous studies have reported the prevalence of these symptoms, our results shed light on the aspects of the disease for which ME/CFS patients believe they require support. The distribution of scores for each item reveals that there is not universal agreement among our respondents about which

\footnotetext{
${ }^{2}$ The high number of female participants reflects the fact that women and men are affected by ME/CFS at a ratio of approximately 5:1 [32]
}

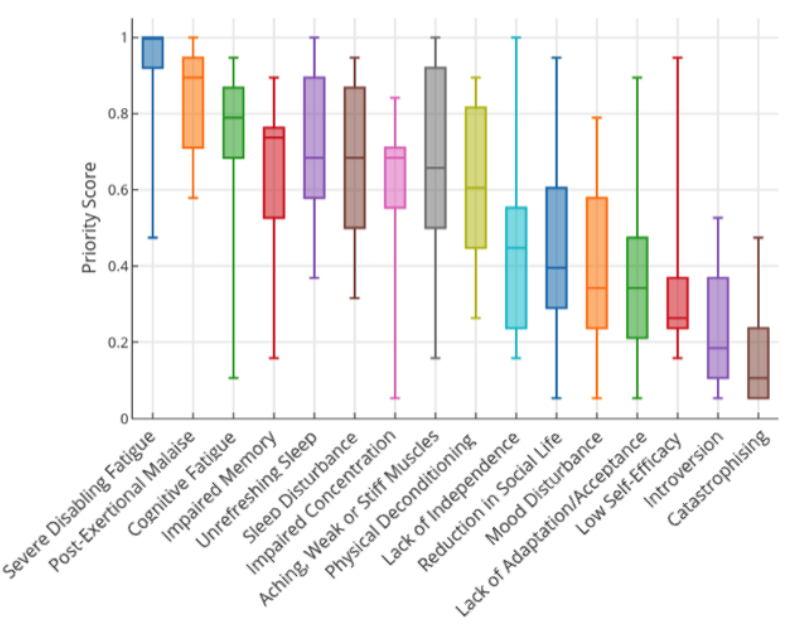

Figure 1: Self-management priority scores assigned to ME/CFS disease symptoms by survey respondents.

aspects of the disease self-management apps should prioritize. However, we observe less variance among the highest and lowest rated items, i.e. there is stronger agreement that selfmanagement technologies should aim to support physical fatigue, and that perpetuating factors such as introversion and catastrophizing are lower priority.

\section{STUDY 2 - DISCUSSION GROUPS: EXISTING SELF-MANAGEMENT PRACTICES}

Our second study builds on Study 1 by investigating ME/CFS patients' existing use of technology for self-managing their condition. Specifically, we explore the technologies they use to support self-management, the benefits and challenges that they have encountered, and their emergent needs for enacting particular practices. To explore these issues we collected qualitative data using three online discussion groups. Two of these were closed ME/CFS support groups on Facebook and one was an online public ME/CFS discussion forum. Previous research indicates that initiating discussions using Facebook is a valid approach to collecting data from hard-to-reach populations $[8,44]$. One researcher, who had actively participated in all three forums for several months in order to become familiar with the community norms and establish rapport with members, acted as facilitator for the discussions.

The researcher initiated a new discussion thread in each group, providing an overview of the research and the topics to be discussed, including details regarding anonymity of comments for research publication. Each post was vetted by community moderators in accordance with their established codes of conduct. Participants were invited to introduce themselves to one another and provide some background about their condition. The researcher posed the following opening questions: "Are there any technologies or applications you have used to help you manage your ME/CFS? Do you think that they 
help? Do you have any particular issues with these devices or applications?". The researcher reassured participants that they were not required to respond quickly and that they could do so in their own time, when they felt able to. The researcher facilitated ongoing discussions by providing prompts for deeper exploration of topics and guiding conversation towards the management of symptoms (from Study 1 ) that were yet to be discussed. Conversations remained open for 6 weeks.

52 individuals contributed to the discussions across the three groups (Facebook Group 1: N=17, Facebook Group 2: $\mathrm{N}=22$, Discussion Forum Group: $\mathrm{N}=13$ ). This comprised all participants from Study 1 and an additional 13 people who did not take the Study 1 survey. Participants posted the following number of responses in each group - FB Group 1: Mean $=6.41$, S.D. $=3.55 ;$ FB Group 2: Mean $=2.67$, S.D. $=2.17$; Discussion Forum Group: Mean = 3.62, S.D. $=2.05$.

All participant comments were analyzed using a general inductive approach [69]. Respondent names were replaced with random IDs prior to analysis. Two authors engaged in close reading of responses and identified data relevant to understanding: (1) participants' reported practices for selfmanagement; (2) the technological interventions used to perform self-management; and (3) perceived strengths and weaknesses of these technologies. These authors developed initial themes through inductive coding. We then conducted a coding consistency check [69] in which the third author applied these themes to the data as a test of credibility and dependability, and to support theme refinement.

\section{STUDY 2 FINDINGS}

\section{Self-Management Practices and Technology Use}

Participants described engaging in data collection to support three kinds of self-management relevant to ME/CFS. These were activity pacing and understanding healthy limits, supporting routinization, and coping and supporting mental health. Participants reported using a variety of commercial self-tracking devices and smartphone applications to achieve these goals. However, only one participant reported using an app designed specifically for management of ME/CFS ('ME/CFS Diary').

The most commonly used technologies were wearable tracking devices (e.g. Fitbit, Garmin Vivosmart, Polar heartrate monitors, Apple Watch) and general-purpose (i.e. nonME/CFS specific) health and wellbeing tracking apps such as MyFitnessPal, Google Fit and Apple Health. These were used to capture data related to: physical activity/exercise, sleep, heart rate and $\mathrm{HR}$ variability, activity type, duration and intensity, diet/calorie consumption, menstrual cycles, stress levels, blood pressure and other primary symptoms.

Activity Pacing and Understanding Healthy Limits. Participants described attempting to use their tracked data to support activity pacing and energy preservation techniques such as spoon management [50] and energy envelope maintenance [34]. "Pacing is such a big deal" (P50), "Pacing is a big deal for me too" (P15). Some had attempted to assess their energy expenditure and inform their pacing strategies by combining real-time monitoring of their physiological data (e.g. step counts, heartrates) with records of their activities: "I found it [a heart-rate monitor] useful in helping me to find and stay within my energy window." (P27). One person had used such data for "choosing which self-care acts to use my energy for on any given day. Do I shower or eat? Do I take my vitamins or clip my nails?” (P1).

Many participants described their attempts to prevent PEM and severe disabling fatigue through the use of physical activity trackers. However, these tools were not used for increasing physical activity or exceeding a particular target (e.g. 10,000 steps per day [45]), but were instead appropriated as a way of regulating activity and identifying thresholds to be avoided: "I use my steps counter in reverse - to help me set limits on the number of steps I take so I am not suffering so much pain and fatigue the next day... through experience I have found my optimal number of steps each day is 2,000 to 4,000" (P11), "My Apple Watch tracks all of my motion/activity, standing, and heart rate. I set a goal (a threshold for me to trigger a crash) and watch it during the day" (P30).

Understanding healthy limits was an important goal for our participants, and one individual had used the results of specialized medical testing to support this goal. He made use of a wearable device and smartphone app to monitor how close he came to overexertion: "I have cardiopulmonary exercise test results that objectively show when I go from my aerobic to anaerobic threshold, is the point where I need to stop. My HR was 100 at that point. So, my goal is to keep my HR from going above 100 BPM." (P49). At the same time, there was a need to engage in a baseline level of activity (an amount that can be performed without experiencing PEM) so as to avoid the onset of muscle weakening and deconditioning. However, one participant reported that it was difficult to identify her 'baseline' activity level, given daily fluctuations in her condition.

Some participants had attempted to monitor their symptoms and record information that might lead to the identification of triggers. This was seen as a way of understanding their condition: "I keep a graph of how many hours of activity I've done (which is great for showing how well I have kept to my baseline) and I attempt to monitor how my diet, self-discipline, emotional stress, mind and body rests etc affect it" (P21), "I need to be able to track my symptoms to figure out the contexts in which I'm getting better or worse" (P25).

Supporting Routinization. Another way of supporting selfmanagement of ME/CFS was through activity planning and scheduling in pursuit of routinization $[6,40]$. This was typically done through the use of apps that incorporated calendars, 
to-do lists, reminders and alarms. These features were used to establish routines according to fluctuations in energy, and to balance activities that involved either physical or cognitive demands. These features were also used to support memory when experiencing impaired cognitive function: "I need reminders as I forget everything" (P23). "Ijust can't be without my calendar on my phone" (P50), "My iPad and iPhone are booster packs for my brain" (P11). One participant (P21) reported using a tailored ME/CFS diary app to support scheduling of tasks but gave negative feedback about the app because it enforced recording of activities in 30-minute timeslots. This was problematic because her condition rarely allowed her to do any single activity for an entire 30-minute period.

Some people found that routinization was bolstered by gamification mechanics that helped to form habits. One participant reported using an application called 'Habitica', which incorporates gamified self-management features: "it has a habits list, a daily list and a to-do list. It has an avatar and as you check off things or tick the habits you're trying to establish you level up and gain items. This helps me acknowledge how well I'm taking care of myself. You also lose health for bad habits and regain it when you level up. It's quite a fun way to gauge what I'm actually doing. Plus, I love my purple tiger [avatar] that's now big enough to ride" (P8). P11 thought that gamification could be used effectively to help them to stay within their energy envelope: "Would love a points system that rewards all self-care initiatives... It should also give reward points for setting healthy limits [for] the number or length of activities to prevent overexertion."

Coping and Supporting Mental Health. Finally, people spoke about their practices related to their mental health, including accepting their disease and preventing catastrophizing. P34 felt that an important aspect of her self-management was "coming to terms with the illness" and "accepting what has happened". Two participants used mobile app-based meditation exercises to manage their worries and stress: "A challenge is coping with the sinking feeling that I can't do any of the things I want or need to do. Meditation has really helped and I have learnt to not let worry make things worse" (P12). P11 reported engaging in audio-guided relaxation exercises, using a Muse headband (an EEG-based neurofeedback device) to "deepen my daily meditation practice... I'm very grateful for these devices". Practices relating to mental wellbeing were sometimes interwoven with the management of energy expenditure. P11 described using mindfulness techniques as a way to "check in with my body" and guarantee a moment of mental pause to reflect on her physical and cognitive fatigue.

\section{Problems with Self-Management Using Technology}

Participants described a range of issues that they encountered when engaging in self-management. We delineated these into technology-centered issues, which relate to general usability difficulties or features that do not accommodate the needs of $\mathrm{ME} / \mathrm{CFS}$ patients, and condition-centered issues, which relate to self-management challenges that people with ME/CFS face as a result of their condition, rather than as a result of design choices.

Technology-Centered Issues. Many participants stated their interest in using technologies to support activity pacing and described appropriating commercial 'self-tracking' solutions in pursuit of this. At the same time, participants were quick to note the limitations of these technologies for pacing. Specific issues included an over-emphasis on increasing physical activity, limited ways of capturing different kinds of exertion, and a lack of support for alerts about over-activity.

Some participants described how self-management with commercial devices such as Fitbit was difficult because these devices are not designed around ME/CFS. They desired tailored solutions and were cautious about using generic health applications: "We need devices that are suitable for monitoring the signs of CFS and whose data will be properly interpreted in terms of what those signs and symptoms mean about our conditions. It will be important to avoid misdirection when something for one condition is used for a very dissimilar one." (P24).

Participants wanted features that would help them to avoid going over their activity threshold: "I've wanted a simple way of triggering an alarm when you go over your limit. The commercially available devices are all aimed at healthy people trying to improve their fitness and they're incredibly complicated to program just to get that alarm for an upper limit. As a brainfogged person with ME, I found them impossible." (P9).

A further limitation was that participants found it difficult to track 'fatigue' as a general concept: "I'd love [a tracker] more directly related to fatigue" (P36), "I discovered FibroMapp, but it doesn't give me many fatigue-related specifics, and doesn't let me enter fatigue more than once a day... Fatigue is kinda hard to measure" (P26). They also noted the limited availability of tools to support tracking of the cognitive aspects of their disease, such as mental exertion, compared with those for tracking and monitoring physical activity. "The cognitive burden should really feature." (P31). "[I need] something that lets me keep track of sedentary activities and emotional events in terms of energy use." (P2). "I don't generally use the steps as a guide for when to rest as I get really fatigued from mental exertion too - driving, reading etc.” (P15).

Participants were concerned about the accuracy of technologies like Fitbit: "I don't believe the readings are accurate." (P24). While the accuracy of step counters is a common complaint among users [77], our participants focused more on the trackers' inaccuracy in terms of differentiating the intensity of activity: "I find the Fitbit largely inaccurate in figuring out strenuous versus light activity" (P3). This was seen as a concern when attempting to 'pace' one's energy expenditure. 
One person described her attempts to stay within her energy envelope by using a heart rate monitor: "I guess I don't bother with exactly how high it is, just work to keep it under a level where I 'feel' it' (P36).

Beyond problems with activity pacing, many participants highlighted a need for greater simplicity in the tools they used for tracking data. Some participants had felt overwhelmed when attempting to capture data related to different facets of their condition: "I get overwhelmed by having to track a bunch of symptoms every day" (P36), "I have always struggled with keeping records/journal for tracking symptoms. I get bogged down in it, because there are so many variables" (P21). This general notion of tracking burden has been previously reported in the literature on personal informatics [11, 13, 37], but was particularly acute for our participants given the specifics of their condition.

Condition-Centered Issues. In addition to issues with selfmanagement technologies, participants discussed how living with ME/CFS made it difficult to maintain self-management practices. For example, many identified activity pacing as one of the most significant self-management challenges but stated that their ability to engage in pacing was often hampered by their symptoms: "Pacing is my number one struggle... hindered by my mental fatigue and brain fog" (P21), "I agree with everyone else here that pacing is the number one challenge. I'm trying to establish a habit of checking in with my body three or four times a day to see how I'm doing and whether I need to reassess my goals with the energy I have left... before overdoing it and suffering the consequences" (P11).

Two participants reported that they relied on others to track and monitor their symptoms as result of the difficulties they faced with self-management applications. Previous work has noted that self-care is often a collaborative endeavor [18, 55] and this was reflected in some of our participants' reliance on family members for self-management. For example, "I keep in touch with my son during the day as he monitors my daily ups and downs." (P41). "I utilize my husband [for monitoring energy levels] as much as possible when he's at home." (P32).

Incidents of 'crashing' (suffering PEM) also posed a significant challenge for those engaging in self-management. One participant commented "Crashing leaves me feeling particularly ill and I find it difficult to keep my eyes open even" (P3). The consequence of this was that participants would incur lapses in recording data that coincided with 'crash days'. For example, P50 reported difficulties with recording data during bouts of severe brain fog and migraines: "When I record a migraine my app will lead me to several different pages to annotate each and every little thing. That would be good, but during a migraine, I simply want to go lay down." Participants discussed wanting to record what was happening in the moment to avoid problems with recall and retrospective logging (exacerbated by disease related memory impairments), but revealed that reminders were often too late or were delivered when symptoms remained obtrusive.

\section{STUDY 3 - USER STUDY: EXPERIENCES WITH SELF-MANAGEMENT APPS}

Study 2 demonstrated that people with ME/CFS patients leverage self-tracking devices and smartphone apps to support self-management of their disease. Our third study provides further understanding of ME/CFS patients' perspectives on the use of smartphone applications to support management of key disease symptoms (identified in Study 1).

We conducted a user study in which 24 people with ME/CFS used a series of self-management apps. All of these individuals had also participated in Studies 1 and 2. We selected three apps spanning the range of symptoms that participants had previously prioritized in Study 1, and the range of self-management approaches identified in Study 2 (e.g. self-tracking, scheduling, automated and manual data logging, gamification, mindfulness, etc.). These apps were not provided in order to assess the efficacy of app-based interventions, but rather to provide prompts to elicit further feedback and design requirements for ME/CFS self-management technologies.

We compiled a list of all apps that participants had reported using in Study 2, combined with additional Google Play and Apple App Store self-management apps related to ME/CFS disease symptoms. We then applied the PRISMA protocol [5] for shortlisting apps for evaluation. This comprised: filtering of duplicate apps; eliminating non-English apps; removing apps requiring purchase and those not updated within the past year; and separation of apps into their core functionality (e.g. separating apps designed for diagnosis, and those for ongoing self-management). We also conducted literature reviews to identify shortlisted apps whose effectiveness had been demonstrated for general health and wellbeing management. From our final shortlist we selected three self-management apps to deploy in our study.

App 1: Sleep-2-Peak (S2P) objectively measures and tracks fluctuations in fatigue [9]. S2P incorporates 3-minute Psychomotor Vigilance Tests (reaction time tests), which accurately measure fatigue-related changes in physical and mental performance [9]. The app then recommends optimal sleep/wake times to the user based on reaction time patterns. S2P also enables users to investigate the association between fatigue and substances such as caffeine and medications, through self-logging features. S2P provided participants with features to track a validated measure of fatigue based on reaction times [9], to reflect on their physical/mental performance and sleep-cycle, and to identify potential factors that may influence their symptoms. We selected S2P because it supports the monitoring of fatigue, which was the most important 
disease symptom identified in Study 1 . In addition, Study 2 revealed that people with ME/CFS currently try to track fatigue with devices like Fitbit, but find these solutions unsuitable.

App 2: SuperBetter (SB) gamifies real-life tasks and behaviors in order to promote the achievement of personal goals, referred to as "Power-ups" (e.g. walking a certain distance, stretching, taking a moment to rest) and the avoidance of barriers to goals, labelled as "Bad Guys" (e.g. over-exerting oneself, eating unhealthily) [14]. Users can select predefined power-ups and bad-guys to target or avoid, and invite other users with similar experiences ("Allies") to help them define their own. SB provided our participants with opportunities to gamify existing self-management practices, as well as find new activities that may build physical, mental, emotional and social resilience. Randomized controlled trials have demonstrated that SB is an effective self-management tool for treating depressive symptoms [63], but no studies have explored its use for managing ME/CFS specifically.

We selected SB because it gives users the opportunity to support routinization through habit-formation and gamification, which Study 2 revealed as beneficial for people with ME/CFS. The app also enables user customization of goals to support aspects of physical and mental wellbeing that matter most to individuals.

App 3: Smiling Mind (SM) provides guided meditations and exercises based on mindfulness practices [47]. Mindfulness has been identified as beneficial for ME/CFS patients in terms of managing impairment, depressed mood, and catastrophic thinking [62]. Mindfulness was used by some Study 2 participants as a technique for pausing to 'check in' with their body (e.g. for pacing) and to manage mental wellbeing. SM also includes a range of additional features, e.g. timers, reminders and tracking of time spent on mindfulness activities, which may support routinization and scheduling.

All 24 user study participants had received an ME/CFS diagnosis from a medical professional. The average age of participants was $40.1 \mathrm{yrs}$ (Range $=20-64$, S.D. $=11.3 \mathrm{yrs}$ ). 21 identified as female and 3 identified as male. 12 were residents of the United Kingdom; 7 USA; 3 Australia; 2 Canada. Participation involved using up to two of the selected apps (randomized selection) at least once per day for a period of two weeks, followed by completion of an online feedback form that captured details of users' experiences with each app ${ }^{3}$. Participants were asked to describe how they used the application, if there was anything they particularly liked/disliked, and any benefits/limitations that the app had for management of their condition. In line with our general approach for involving people with ME/CFS in our research, we provided detailed task instructions and regular reminders throughout the two-week

\footnotetext{
${ }^{3} 12$ participants opted to use two apps, the other 12 used a single app. $\mathrm{N}=12$ for each app.
}

period. Participants were instructed to suspend their use of apps if they made their symptoms worse. We also provided flexible deadlines for returning feedback. This was important as the nature of the participants' ME/CFS meant that several people required additional time, due to adverse health.

As with Study 2, feedback from participants was analyzed by the first and second authors using a general inductive approach [69], with coding consistency checks and theme refinement conducted by the third author.

\section{STUDY 3 FINDINGS}

We detail participants' responses according to positive and negative aspects of the apps for self-management of ME/CFS.

\section{Positive App Experiences}

Participants reported that some in-app features for tracking data about their symptoms, behaviors and self-management activities provided value for supporting pacing and routinization. For example, Participants 8 and 51 reflected on their fatigue levels using data visualizations and diary views in S2P. Participant 34 used records of her SB activities (i.e. completed and outstanding power-ups/bad-guys) "as a tool to see how I had done during the day" and to schedule further tasks and inform her activity pacing decisions.

Participant 31 explained that her S2P data accurately reflected her fatigue patterns, around which she structured her routine: "It shows I am more alert in the mornings and then fade throughout the day. By evening, my mind is not very clear". She felt reassurance in seeing these patterns confirmed by her data, but believed that more beneficial insights might come from exploring how her fatigue changed in response to certain events or triggers: "I did not have any sleepless nights during this period, but I'd like to test that out". P27 described the value of having digital records of her activities and fatigue, not for personal reflection, but for sharing with health professionals: "It could serve as proof to doubting doctors".

Two participants reported that the gamification elements of SB raised their enthusiasm for self-management: "Iliked the game aspect, that approach appeals to me and would encourage me to engage" (P44), "Managing my CFS is so much work, making some of that fun is good" (P34). Three people valued the ability to define their own behaviors to target or avoid in SB: "I liked the simplicity and that I could make my own to personalize it to $m e$ " (P7). Participant 24 enjoyed finding power-ups contributed by other users, which were appropriate for her condition: "I liked the reminder to drink water and some of the power-ups like hugging yourself, dancing or being silly".

Many participants valued opportunities for low-effort interactions with the apps, for example receiving audio instructions for guided relaxation and mindfulness exercises in SM, without the need to hold the phone or view the screen: "It required less energy expenditure to participate... the voices were 
gentle and soothing" (P44). Participant 19 wanted to see this approach incorporated into other apps in order to reduce interaction with the screen: "I'd like an option of having the goals read out loud".

In terms of addressing symptoms, SM's mindfulness exercises provided benefits to participants for mental, emotional, cognitive and sleep-related disease factors: "I have a lot of anxiety surrounding my symptoms and I believe this app helped that. Ido think it is a great tool to have to at least help with anxiety and possibly with some cognitive issues." (P14), "It helped me calm my thoughts before I went to sleep... I was less anxious and restless. I really like the app and am going to continue to use it" (P34).

\section{Negative App Experiences}

Participants using SB reported that the activity goals represented by power-ups, which were described within the app as being beneficial for physical, mental and social resilience, were often at odds with their condition. Some were frustrated when encountering suggestions for physically and cognitively demanding activities that were beyond their capabilities: "Most of the activities were at odds with having CFS" (P44), "There were suggestions to walk around the block etc. that are beyond my limitations. There was also a lot to do with motivation to do a thing, whereas I need more motivation to slow down and rest" (P19).

Only a few participants made use of the ability to create their own power-ups and bad guys in SB. This was either due to difficulties in setting appropriate goals and barriers, because of limited understanding of their own limits, e.g. "I found the power-ups and bad guys easy to use, but more difficult to think up my own" (P7), or as a result of difficulties with regard to the complexity of the app interface: "I need a simpler interface... Have everything on one page, make it as easy as possible to add own goals on the same page instead of in a menu" (P19).

Some participants reported that cognitive fatigue and impaired concentration made it difficult to use some of the apps. Three participants found the app navigation in SB and SM to be significant barriers and reduced their use of the apps as a result: SB: "It's too overcomplicated. The layout and functionality was too overwhelming to use with ease, especially if ill or experiencing brain fog." (P7). "Writing was quite small in some areas and I struggled to concentrate on reading it." (P13), SM: "interface too busy...caused brain fog" (P39).

Other symptoms such as muscle weakness and physical fatigue also had an effect on app use. Participant 27 reported that the recommended posture for holding the phone during the $\mathrm{S} 2 \mathrm{P}$ reaction time tests was uncomfortable, but was unsure whether altering this position would affect the accuracy of test results. In contrast, Participant 13 appreciated that each of the apps were suitable for brief interactions: "short enough that my muscles didn't seize from holding the phone".
Finally, one participant described terminology, such as the 'Lazy Exercise' label given to a set of power-ups in SB, as "very disheartening and shaming for those with more severe CFS... I felt awful after being confronted with this... lazy is so often thrown at CFS sufferers as an insult. To not even be capable [of these tasks] was horrible. It deterred me so much I stopped using the app." (P44).

\section{DISCUSSION AND DESIGN IMPLICATIONS}

This work contributes a deeper understanding of ME/CFS patients' needs for self-management technologies. The studies also affirm the results of prior work [11,56], which has highlighted that people with chronic conditions encounter significant challenges when self-management tools are not tailored to the specifics of their condition. Our studies have revealed a set of particular concerns that are relevant to the self-management of ME/CFS, from which we propose five high-level design recommendations.

1. Help to determine personal thresholds. Our research suggests that people with ME/CFS want to self-manage their physical and cognitive energy expenditure by determining personalized activity thresholds. Specifically, Study 2 showed that patients wanted to identify a minimum daily activity threshold to avoid problems such as muscle deconditioning, and yet also wanted to identify a maximum daily threshold to avoid feelings of severe disabling fatigue and instances of PEM. Understanding these thresholds is also a key precursor to activity pacing and staying within an 'energy envelope'. However, participants reported that their current solutions (commercial self-tracking technologies such as step counters and heart rate monitors) do not have explicit support for identifying and recording these thresholds. This may be because these devices are designed with the aim of simply increasing physical activity [e.g. 16], which does not adequately reflect the self-management goals of people living with ME/CFS.

To address this, future designs should provide features that allow people with ME/CFS to determine appropriate thresholds for both physical and mental activity. This will support the recognition of activity levels that cause crashes, help to avoid instances of PEM, and can also support the budgeting of energy expenditure.

2. Provide support for activity pacing and routinization. Our research suggests that managing symptoms related to physical fatigue is a priority for people with ME/CFS (Study 1) and that this is typically achieved by appropriating commercial activity trackers in service of activity pacing (Study 2). We also found that people with ME/CFS wanted to routinize their daily activities by using timers, calendars, and to-do lists to manage tasks (Studies 2 and 3). However, participants noted that activity trackers (which they used to inform pacing decisions) do not typically include these kinds of functionality. 
To address this, future self-management applications should provide explicit support for activity pacing and should allow for routines to be established and maintained. For example, an application might include energy budgeting tools that help to plan activities, and could issue reminders to ensure that these activities are completed on time. The application could also set appropriate goals given the user's previous energy expenditure and their remaining budget for the day, and could incorporate mechanisms to alert users when they are at risk of exceeding their limits.

3. Create tools to support fatigue tracking. All three of our studies revealed that people with ME/CFS wanted to track and monitor physical and cognitive fatigue as general concepts. However, participants in Study 2 reported finding this hard with most existing technologies.

To address this, future self-management applications could enable tracking of fatigue through self-report mechanisms or semi-automated approaches like the reaction time tests used in Sleep-2-Peak (Study 3). However, these methods of data collection require user effort in order to log data, and Study 3 showed that this effort may be particularly problematic for people with ME/CFS because it can contribute to feelings of fatigue. Future work could therefore explore the potential for fully automated fatigue logging, such as through wearable and 'cognitive personal informatics' devices like EEG [23], or by using patterns of smartphone app use to infer a user's state [53].

4. Motivate users to engage in self-management. Study 3 showed that people with ME/CFS valued features that motivated them to continue using a technology, and which encouraged them to track data. Gamification was cited as an effective way of achieving continued use because it introduced an element of fun to self-management. In line with prior work, which demonstrates the potential of gamification to facilitate self-management in persons with chronic conditions (e.g. [49]), our findings suggest that self-management technologies for ME/CFS should employ lightweight gamification mechanisms as a way of fostering engagement and continued motivation.

However, the nature of ME/CFS means that it may be difficult to use self-management technologies during symptom flares. Therefore, we suggest that gamification mechanisms for ME/CFS should be designed to reward cumulative selfmanagement activity, and should avoid penalizing people if they incur periods of inactivity (such as resetting progress or changing the interface if certain goals are not met [e.g. 17]).

Additionally, designers should be aware of the terminology they use when trying to support self-management. In Study 3, we found that some people with ME/CFS felt that self-tracking technologies could 'shame' them through accusations of laziness and other prompts that highlight their inability to perform exercise. Feelings of illness-invalidation (such as by associating severe fatigue with laziness) have been shown to be common amongst ME/CFS patients [73]. Experiences of delegitimation and shaming can have further negative consequences, such as by causing patients to isolate from their social circles [15]. This emphasizes the need for a careful and compassionate approach when designing self-management technologies for this population.

5. Tailor support according to individual differences and disease fluctuations. While our studies have drawn attention to key goals and challenges faced by people with $\mathrm{ME} / \mathrm{CFS}$, it is also important to recognise that the severity of particular symptoms can vary from person to person. This was partly evidenced by the findings of Study 1, where symptoms such as Cognitive Fatigue, Impaired Concentration, and Aching Muscles had high variance in their priority scores. This suggests that each individual may be affected by certain symptoms more or less severely. Likewise, it is also important to note that the severity of ME/CFS for a given person can fluctuate on a daily basis. Many of our Study 2 participants described a lack of consistency in their symptoms from one day to the next, a phenomenon that has been observed in other chronic conditions $[12,56]$.

To address this, designers should ensure that technologies can accommodate individual differences between people with ME/CFS. For example, features for activity pacing should be tailorable to the specifics of an individual's condition, such as by allowing adjustable energy envelopes [34]. Self-management technologies should also accommodate the differences that can arise as a result of daily fluctuations in disease activity, perhaps by recognizing 'good days' and 'bad days' in their functionality [12]. For example, users could explicitly disable motivational prompts on bad days, or could receive recommendations for more demanding selfmanagement tasks on better days. Similar guidelines have been proposed for apps to help people with Parkinson's Disease [56], suggesting that this strategy may benefit a range of chronic health conditions.

Finally, we suggest that self-management technologies for ME/CFS should be very simple to use. This is particularly acute for people with ME/CFS because the app itself could act as a symptom trigger if it requires significant cognitive or physical effort to use. People in Study 3 experienced tiredness as a result of complicated menus or hard-to-read fonts, suggesting that designers should pay extra care to user effort when designing technologies for people with ME/CFS.

\section{CONCLUSION AND FUTURE WORK}

This paper has explored the design of self-management technologies for people living with ME/CFS. Study 1 identified a list of symptoms prioritized by people with ME/CFS, giving immediate direction for the design of condition-specific support. Study 2 identified a need for solutions that assist existing self-management practices such as activity pacing 
and fatigue monitoring. Study 3 revealed specific positive and negative design features through participants' responses to a series of commercial apps. Collectively, our studies illustrate the specific needs of people with ME/CFS and provide further evidence that chronic conditions bring unique concerns that must influence technology design $[11,56]$.

Based on our study, we see several opportunities for future work. First, our recommendations can support the design of tailored self-management applications that should be tested in conjunction with ME/CFS patients, such that they have an ongoing stake in the design process [42]. Second, our design recommendations are based on patients' needs and existing practices. However, it is important to recognise that our work does not incorporate the perspectives of clinicians, and these may be complementary in terms of understanding opportunities for self-management [48]. Finally, there may be opportunities to explore how technologies could support the collaboration that occurs between ME/CFS patients and their caregivers [55]. A number of participants in Study 2 acknowledged that they relied on family members for additional support. Future work could explore the design of collaborative applications that provide caregivers with a stake in the condition management process.

\section{REFERENCES}

[1] Leslie A Aaron, Mary M Burke, and Dedra Buchwald. 2000. Overlapping conditions among patients with chronic fatigue syndrome, fibromyalgia, and temporomandibular disorder. Archives of internal medicine 160, 2 (2000), 221-227.

[2] Susan E Abbey. 1996. Psychiatric diagnostic overlap in chronic fatigue syndrome. Chronic Fatigue Syndrome: An Integrated Approach to Evaluation and Treatment (1996), 49-71.

[3] Phil Adams, Elizabeth L. Murnane, Michael Elfenbein, Elaine Wethington, and Geri Gay. 2017. Supporting the Self-Management of Chronic Pain Conditions with Tailored Momentary Self-Assessments. In Proceedings of the 2017 CHI Conference on Human Factors in Computing Systems (CHI '17). ACM, New York, NY, USA, 1065-1077. https://doi.org/10.1145/3025453.3025832

[4] Niloofar Afari and Dedra Buchwald. 2003. Chronic fatigue syndrome: a review. American fournal of Psychiatry 160, 2 (2003), 221-236.

[5] Kevin Anderson, Oksana Burford, and Lynne Emmerton. 2016. App Chronic Disease Checklist: Protocol to Evaluate Mobile Apps for Chronic Disease Self-Management. FMIR Res Protoc 5, 4 (04 Nov 2016), e204. https://doi.org/10.2196/resprot.6194

[6] Deborah Antcliff, Philip Keeley, Malcolm Campbell, Steve Woby, AnneMaree Keenan, and Linda McGowan. 2018. Activity pacing: moving beyond taking breaks and slowing down. Quality of Life Research 27, 7 (01 Jul 2018), 1933-1935. https://doi.org/10.1007/s11136-018-1794-7

[7] Julie Barlow, Chris Wright, Janice Sheasby, Andy Turner, and Jenny Hainsworth. 2002. Self-management approaches for people with chronic conditions: a review. Patient education and counseling 48, 2 (2002), 177-187.

[8] Narelle Biedermann. 2018. The use of Facebook for virtual asynchronous focus groups in qualitative research. Contemporary nurse 54, 1 (2018), 26-34.

[9] Jean-François Brunet, Dominique Dagenais, Marc Therrien, Daniel Gartenberg, and Geneviève Forest. 2017. Validation of sleep-2-Peak:
A smartphone application that can detect fatigue-related changes in reaction times during sleep deprivation. Behavior Research Methods 49, 4 (01 Aug 2017), 1460-1469. https://doi.org/10.3758/s13428-016-0802-5

[10] Davis Buls and John Rooksby. 2017. Technology for Self-Management of Rosacea: A Survey and Field Trial. In Proceedings of the 2017 CHI Conference Extended Abstracts on Human Factors in Computing Systems (CHI EA '17). ACM, New York, NY, USA, 1516-1523. https://doi.org/10.1145/3027063.3053080

[11] Adrian Bussone, Simone Stumpf, and George Buchanan. 2016. It Feels Like I'm Managing Myself: HIV+ People Tracking Their Personal Health Information. In Proceedings of the 9th Nordic Conference on Human-Computer Interaction (NordiCHI '16). ACM, New York, NY, USA, Article 55, 10 pages. https://doi.org/10.1145/2971485.2971542

[12] Kathy Charmaz. 1993. Good days, bad days: The self in chronic illness and time. Rutgers University Press.

[13] Eun Kyoung Choe, Nicole B. Lee, Bongshin Lee, Wanda Pratt, and Julie A. Kientz. 2014. Understanding Quantified-selfers' Practices in Collecting and Exploring Personal Data. In Proceedings of the SIGCHI Conference on Human Factors in Computing Systems (CHI '14). ACM, New York, NY, USA, 1143-1152. https://doi.org/10.1145/2556288.2557372

[14] Tommy Chou, Laura J. Bry, and Jonathan S. Comer. 2017. Multimedia Field Test: Evaluating the Creative Ambitions of SuperBetter and Its Quest to Gamify Mental Health. Cognitive and Behavioral Practice 24, 1 (2017), 115 - 120. https://doi.org/10.1016/j.cbpra.2016.10.002

[15] Juanne N Clarke and Susan James. 2003. The radicalized self: the impact on the self of the contested nature of the diagnosis of chronic fatigue syndrome. Social Science \& Medicine 57, 8 (2003), 1387-1395.

[16] Sunny Consolvo, Katherine Everitt, Ian Smith, and James A. Landay. 2006. Design Requirements for Technologies That Encourage Physical Activity. In Proceedings of the SIGCHI Conference on Human Factors in Computing Systems (CHI '06). ACM, New York, NY, USA, 457-466. https://doi.org/10.1145/1124772.1124840

[17] Sunny Consolvo, David W. McDonald, Tammy Toscos, Mike Y. Chen, Jon Froehlich, Beverly Harrison, Predrag Klasnja, Anthony LaMarca, Louis LeGrand, Ryan Libby, Ian Smith, and James A. Landay. 2008. Activity Sensing in the Wild: A Field Trial of Ubifit Garden. In Proceedings of the SIGCHI Conference on Human Factors in Computing Systems (CHI '08). ACM, New York, NY, USA, 1797-1806. https://doi.org/10.1145/1357054.1357335

[18] Juliet Corbin and Anselm Strauss. 1985. Managing chronic illness at home: three lines of work. Qualitative Sociology 8, 3 (1985), 224-247.

[19] Todd E Davenport, Staci R Stevens, J Mark VanNess, Jared Stevens, and Christopher R Snell. 2018. Checking our blind spots: current status of research evidence summaries in ME/CFS. British fournal of Sports Medicine (2018). https://doi.org/10.1136/bjsports-2018-099553 arXiv:https://bjsm.bmj.com/content/early/2018/07/17/bjsports-2018099553.full.pdf

[20] Wilfred C. de Vega, Santiago Herrera, Suzanne D. Vernon, and Patrick O. McGowan. 2017. Epigenetic modifications and glucocorticoid sensitivity in Myalgic Encephalomyelitis/Chronic Fatigue Syndrome (ME/CFS). BMC Medical Genomics 10, 1 (23 Feb 2017), 11. https://doi.org/10.1186/s12920-017-0248-3

[21] Adrienne. Dellwo. 2018. Myalgic Encephalomyelitis or Chronic Fatigue Syndrome. Available Online. https: //www.verywellhealth.com/myalgic-encephalomyelitis-me-715663

[22] Carl DiSalvo, Jonathan Lukens, Thomas Lodato, Tom Jenkins, and Tanyoung Kim. 2014. Making Public Things: How HCI Design Can Express Matters of Concern. In Proceedings of the SIGCHI Conference on Human Factors in Computing Systems (CHI '14). ACM, New York, NY, USA, 2397-2406. https://doi.org/10.1145/2556288.2557359 
[23] Cillian Dudley and Simon L. Jones. 2018. Fitbit for the Mind?: An Exploratory Study of 'Cognitive Personal Informatics'. In Extended Abstracts of the 2018 CHI Conference on Human Factors in Computing Systems (CHI EA '18). ACM, New York, NY, USA, Article LBW542, 6 pages. https://doi.org/10.1145/3170427.3188530

[24] Melissa Edmonds, Hugh McGuire, and Jonathan R Price. 2004. Exercise therapy for chronic fatigue syndrome. Cochrane database of systematic reviews 3 (2004).

[25] Emerge Australia. 2019. Managing Symptoms on a Daily Basis (webpage). https://emerge.org.au/diagnosis/ managing-symptoms-daily-basis/

[26] Michael Falk Hvidberg, Louse Schouborg Brinth, Anne V. Olesen, Karin D. Petersen, and Lars Ehlers. 2018. The Health-Related Quality of Life for Patients with Myalgic Encephalomyelitis / Chronic Fatigue Syndrome (ME/CFS). PLoS ONE 10, 7 (2018), e0132421. https://doi.org/10.1371/journal.pone.0132421

[27] Sergio Felipe, Aneesha Singh, Caroline Bradley, Amanda C. de C. Williams, and Nadia Bianchi-Berthouze. 2015. Roles for personal informatics in chronic pain. In Pervasive Computing Technologies for Healthcare (PervasiveHealth), 2015 9th International Conference on. IEEE, 161-168.

[28] Keiji Fukuda, Stephen E Straus, Ian Hickie, Michael C Sharpe, James G Dobbins, and Anthony Komaroff. 1994. The chronic fatigue syndrome: a comprehensive approach to its definition and study. Annals of Internal Medicine 121, 12 (1994), 953-959.

[29] Keith Geraghty, Mark Hann, and Stoyan Kurtev. 2017. Myalgic encephalomyelitis/chronic fatigue syndrome patients' reports of symptom changes following cognitive behavioural therapy, graded exercise therapy and pacing treatments: Analysis of a primary survey compared with secondary surveys. Fournal of Health Psychology 0,0 (2017), 1359105317726152. https://doi.org/10.1177/1359105317726152 arXiv:https://doi.org/10.1177/1359105317726152 PMID: 28847166.

[30] Ellen M Goudsmit, Jo Nijs, Leonard A Jason, and Karen E Wallman. 2012. Pacing as a strategy to improve energy management in myalgic encephalomyelitis/chronic fatigue syndrome: a consensus document. Disability and rehabilitation 34, 13 (2012), 1140-1147.

[31] Stephen T Holgate, Anthony L Komaroff, Dennis Mangan, and Simon Wessely. 2011. Chronic fatigue syndrome: understanding a complex illness. Nature Reviews Neuroscience 12, 9 (2011), 539.

[32] Leonard Jason, Susan Torres-Harding, and Mary Gloria Njoku. 2006. The Face of CFS in the U.S. The CIFIDS Chronicle (01 2006), 16-21.

[33] Leonard A Jason. 2017. The PACE trial missteps on pacing and patient selection. Journal of Health Psychology 22, 9 (2017), 1141-1145.

[34] Leonard A Jason, Molly Brown, Abigail Brown, Meredyth Evans, Samantha Flores, Elisa Grant-Holler, and Madison Sunnquist. 2013. Energy conservation/envelope theory interventions. Fatigue: Biomedicine, Health \& Behavior 1, 1-2 (2013), 27-42.

[35] Leonard A Jason, Meredyth Evans, Suzanna So, Jilian Scott, and Abigail Brown. 2015. Problems in defining post-exertional malaise. fournal of Prevention \& Intervention in the Community 43, 1 (2015), 20-31.

[36] Leonard A Jason, Judith A Richman, Alfred W Rademaker, Karen M Jordan, Audrius V Plioplys, Renee R Taylor, William McCready, Cheng-Fang Huang, and Sigita Plioplys. 1999. A community-based study of chronic fatigue syndrome. Archives of Internal Medicine 159, 18 (1999), 2129-2137.

[37] Simon L. Jones and Ryan Kelly. 2018. Dealing With Information Overload in Multifaceted Personal Informatics Systems. Human-Computer Interaction 33, 1 (2018), 1-48. https://doi.org/10.1080/07370024.2017. 1302334 arXiv:https://doi.org/10.1080/07370024.2017.1302334
[38] Predrag Klasnja and Wanda Pratt. 2012. Healthcare in the pocket: mapping the space of mobile-phone health interventions. Fournal of Biomedical Informatics 45, 1 (2012), 184-198.

[39] Anthony L Komaroff and Dedra Buchwald. 1991. Symptoms and signs of chronic fatigue syndrome. Reviews of Infectious Diseases 13, Supplement_1 (1991), S8-S11.

[40] Daphne Kos, Inge Van Eupen, Jill Meirte, Deborah Van Cauwenbergh, Greta Moorkens, Mira Meeus, and Jo Nijs. 2015. Activity pacing selfmanagement in chronic fatigue syndrome: a randomized controlled trial. American fournal of Occupational Therapy 69, 5 (2015), 6905290020p16905290020p11. https://doi.org/10.5014/ajot.2015.016287

[41] Kyriaki Kouyanou, Charles E Pither, and Simon Wessely. 1997. Iatrogenic factors and chronic pain. Psychosomatic Medicine 59, 6 (1997), 597-604.

[42] Eliana M. Lacerda, Caroline C. Kingdon, Erinna W. Bowman, and Luis Nacul. 2018. Using a participatory approach to develop and implement the UK ME/CFS Biobank. Fatigue: Biomedicine, Health \& Behavior 6, 1 (2018), 1-4. https://doi.org/10.1080/21641846.2018.1396021 arXiv:https://doi.org/10.1080/21641846.2018.1396021

[43] Krupp LB, Sliwinski M, Masur DM, Friedberg F, and Coyle PK. 1994. Cognitive functioning and depression in patients with chronic fatigue syndrome and multiple sclerosis. Archives of Neurology 51, 7 (1994), 705-710. https://doi.org/10.1001/archneur.1994.00540190089021

[44] Anastasia Aldelina Lijadi and Gertina Johanna van Schalkwyk. 2015. Online Facebook focus group research of hard-to-reach participants. International fournal of Qualitative Methods 14, 5 (2015), 1609406915621383.

[45] James J. Lin, Lena Mamykina, Silvia Lindtner, Gregory Delajoux, and Henry B. Strub. 2006. Fish'N'Steps: Encouraging Physical Activity with an Interactive Computer Game. In Proceedings of the 8th International Conference on Ubiquitous Computing (UbiComp'06). Springer-Verlag, Berlin, Heidelberg, 261-278. https://doi.org/10.1007/11853565_16

[46] Lena Mamykina, Elizabeth Mynatt, Patricia Davidson, and Daniel Greenblatt. 2008. MAHI: Investigation of Social Scaffolding for Reflective Thinking in Diabetes Management. In Proceedings of the SIGCHI Conference on Human Factors in Computing Systems (CHI '08). ACM, New York, NY, USA, 477-486. https://doi.org/10.1145/1357054.1357131

[47] Madhavan Mani, David J Kavanagh, Leanne Hides, and Stoyan R Stoyanov. 2015. Review and Evaluation of Mindfulness-Based iPhone Apps. $\quad$ fMIR mHealth uHealth 3, 3 (19 Aug 2015), e82. https://doi.org/10.2196/mhealth.4328

[48] Mollie McKillop, Lena Mamykina, and Noémie Elhadad. 2018. Designing in the Dark: Eliciting Self-tracking Dimensions for Understanding Enigmatic Disease. In Proceedings of the 2018 CHI Conference on Human Factors in Computing Systems (CHI '18). ACM, New York, NY, USA, Article 565, 15 pages. https://doi.org/10.1145/3173574.3174139

[49] Aaron S Miller, Joseph A Cafazzo, and Emily Seto. 2016. A game plan: Gamification design principles in mHealth applications for chronic disease management. Health Informatics fournal 22, 2 (2016), 184-193. https://doi.org/10.1177/1460458214537511 arXiv:https://doi.org/10.1177/1460458214537511 PMID: 24986104.

[50] Christine Miserandino. 2003. The Spoon Theory. Available Online. https://butyoudontlooksick.com/articles/written-by-christine/ the-spoon-theory/

[51] Florian 'Floyd' Mueller, Frank Vetere, Martin R. Gibbs, Jesper Kjeldskov, Sonja Pedell, and Steve Howard. 2005. Hug over a Distance. In CHI '05 Extended Abstracts on Human Factors in Computing Systems (CHI EA '05). ACM, New York, NY, USA, 1673-1676. https://doi.org/10.1145/1056808.1056994

[52] Michael J Muller. 2003. Participatory design: the third space in HCI. Human-computer interaction: Development process 4235 (2003), 165-185. 
[53] Elizabeth L. Murnane, Saeed Abdullah, Mark Matthews, Matthew Kay, Julie A. Kientz, Tanzeem Choudhury, Geri Gay, and Dan Cosley. 2016. Mobile Manifestations of Alertness: Connecting Biological Rhythms with Patterns of Smartphone App Use. In Proceedings of the 18th International Conference on Human-Computer Interaction with Mobile Devices and Services (MobileHCI '16). ACM, New York, NY, USA, 465-477. https://doi.org/10.1145/2935334.2935383

[54] Jo Nijs, Kenny De Meirleir, and William Duquet. 2004. Kinesiophobia in chronic fatigue syndrome: Assessment and associations with disability1. Archives of physical medicine and rehabilitation 85, 10 (2004), 1586-1592.

[55] Francisco Nunes and Geraldine Fitzpatrick. 2015. SelfCare Technologies and Collaboration. International fournal of Human-Computer Interaction 31, 12 (2015), 869-881. https://doi.org/10.1080/10447318.2015.1067498

[56] Francisco Nunes, Paula Alexandra Silva, João Cevada, Ana Correia Barros, and Luís Teixeira. 2016. User interface design guidelines for smartphone applications for people with Parkinson's disease. Universal Access in the Information Society 15, 4 (01 Nov 2016), 659-679. https://doi.org/10.1007/s10209-015-0440-1

[57] Anthony Ocon. 2013. Caught in the thickness of brain fog: exploring the cognitive symptoms of Chronic Fatigue Syndrome. Frontiers in Physiology 4 (2013), 63. https://doi.org/10.3389/fphys.2013.00063

[58] Tom Owen, Jennifer Pearson, Harold Thimbleby, and George Buchanan. 2015. ConCap: Designing to Empower Individual Reflection on Chronic Conditions Using Mobile Apps. In Proceedings of the 17th International Conference on Human-Computer Interaction with Mobile Devices and Services (MobileHCI '15). ACM, New York, NY, USA, 105-114. https://doi.org/10.1145/2785830.2785881

[59] Davy Preuveneers and Yolande Berbers. 2008. Mobile Phones Assisting with Health Self-care: A Diabetes Case Study. In Proceedings of the 10th International Conference on Human Computer Interaction with Mobile Devices and Services (MobileHCI '08). ACM, New York, NY, USA, 177-186. https://doi.org/10.1145/1409240.1409260

[60] Mitchell E Tidy E Price, JR and V Hunot. 2008. Cognitive behaviour therapy for chronic fatigue syndrome in adults. Cochrane Database of Systematic Reviews 3 (2008). https://doi.org/10.1002/14651858.CD001027.pub2

[61] Judith B Prins, Gijs Bleijenberg, Ellen Bazelmans, Lammy D Elving, Theo M de Boo, Johan L Severens, Gert Jan van der Wilt, Philip Spinhoven, and Jos WM Van der Meer. 2001. Cognitive behaviour therapy for chronic fatigue syndrome: a multicentre randomised controlled trial. The Lancet 357, 9259 (2001), 841-847.

[62] Katharine A Rimes and Janet Wingrove. 2013. Mindfulness-based cognitive therapy for people with chronic fatigue syndrome still experiencing excessive fatigue after cognitive behaviour therapy: A pilot randomized study. Clinical Psychology \& Psychotherapy 20, 2 (2013), 107-117.

[63] Ann Marie Roepke, Sara R Jaffee, Olivia M Riffle, Jane McGonigal, Rose Broome, and Bez Maxwell. 2015. Randomized controlled trial of SuperBetter, a smartphone-based/internet-based self-help tool to reduce depressive symptoms. Games for Health fournal 4, 3 (2015), 235-246.

[64] Daniela K. Rosner, Saba Kawas, Wenqi Li, Nicole Tilly, and Yi-Chen Sung. 2016. Out of Time, Out of Place: Reflections on Design Workshops As a Research Method. In Proceedings of the 19th ACM Conference on Computer-Supported Cooperative Work \& Social Computing (CSCW '16). ACM, New York, NY, USA, 1131-1141. https://doi.org/10.1145/2818048.2820021

[65] Jessica Schroeder, Chia-Fang Chung, Daniel A. Epstein, Ravi Karkar, Adele Parsons, Natalia Murinova, James Fogarty, and Sean A. Munson.
2018. Examining Self-Tracking by People with Migraine: Goals, Needs, and Opportunities in a Chronic Health Condition. In Proceedings of the 2018 Designing Interactive Systems Conference (DIS '18). ACM, New York, NY, USA, 135-148. https://doi.org/10.1145/3196709.3196738

[66] Jessica Schroeder, Jane Hoffswell, Chia-Fang Chung, James Fogarty, Sean Munson, and Jasmine Zia. 2017. Supporting Patient-Provider Collaboration to Identify Individual Triggers Using Food and Symptom Journals. In Proceedings of the 2017 ACM Conference on Computer Supported Cooperative Work and Social Computing (CSCW'17). ACM, New York, NY, USA, 1726-1739. https://doi.org/10.1145/2998181.2998276

[67] M.C. Sharpe, L.C. Archard, J.E. Banatvala, L.K. Borysiewicz, A.W. Clare, A. David, R.H. Edwards, K.E. Hawton, H.P. Lambert, and R.J. Lane. 1991. A report-chronic fatigue syndrome: guidelines for research. fournal of the Royal Society of Medicine 84, 2 (1991), 118-121.

[68] Christina Surawy, Jill Roberts, and Amy Silver. 2005. The effect of mindfulness training on mood and measures of fatigue, activity, and quality of life in patients with chronic fatigue syndrome on a hospital waiting list: a series of exploratory studies. Behavioural and Cognitive Psychotherapy 33, 1 (2005), 103-109.

[69] David R. Thomas. 2006. A general inductive approach for analyzing qualitative evaluation data. American fournal of Evaluation 27, 2 (2006), 237-246.

[70] FN Twisk and Michael Maes. 2009. A review on cognitive behavorial therapy (CBT) and graded exercise therapy (GET) in myalgic encephalomyelitis (ME)/chronic fatigue syndrome (CFS): CBT/GET is not only ineffective and not evidence-based, but also potentially harmful for many patients with ME/CFS. Neuro Endocrinology Letters 30, 3 (2009), 284-299.

[71] Boudewijn Van Houdenhove and Patrick Luyten. 2008. Customizing treatment of chronic fatigue syndrome and fibromyalgia: the role of perpetuating factors. Psychosomatics 49, 6 (2008), 470-477.

[72] Jan H. M. M. Vercoulen, Caroline M. A. Swanink, Jan F. M. Fennis, Joep M. D. Galama, Jos W. M. van der Meer, and Gijs Bleijenberg. 1994. Dimensional assessment of chronic fatigue syndrome. fournal of Psychosomatic Research 38, 5 (1994), 383-392.

[73] Norma C Ware. 1992. Suffering and the social construction of illness: The delegitimation of illness experience in chronic fatigue syndrome. Medical Anthropology Quarterly 6, 4 (1992), 347-361.

[74] Carolyn Wilshire, Tom Kindlon, Alem Matthees, and Simon McGrath. 2017. Can patients with chronic fatigue syndrome really recover after graded exercise or cognitive behavioural therapy? A critical commentary and preliminary re-analysis of the PACE trial. Fatigue: Biomedicine, Health \& Behavior 5, 1 (2017), 43-56.

[75] Carolyn E. Wilshire, Tom Kindlon, Robert Courtney, Alem Matthees, David Tuller, Keith Geraghty, and Bruce Levin. 2018. Rethinking the treatment of chronic fatigue syndrome-a reanalysis and evaluation of findings from a recent major trial of graded exercise and CBT. BMC Psychology 6, 1 (22 Mar 2018), 6. https://doi.org/10.1186/s40359-018-0218-3

[76] Anette Winger, Mirjam Ekstedt, Vegard B Wyller, and Sølvi Helseth. 2014. 'Sometimes it feels as if the world goes on without me': Adolescents' experiences of living with chronic fatigue syndrome. Journal of Clinical Nursing 23, 17-18 (2014), 2649-2657.

[77] Rayoung Yang, Eunice Shin, Mark W. Newman, and Mark S. Ackerman. 2015. When Fitness Trackers Don't 'Fit': End-user Difficulties in the Assessment of Personal Tracking Device Accuracy. In Proceedings of the 2015 ACM International foint Conference on Pervasive and Ubiquitous Computing (UbiComp '15). ACM, New York, NY, USA, 623-634. https://doi.org/10.1145/2750858.2804269 